Revista de la red interuniversitaria de estudios sobre las literaturas rioplatenses contemporáneas en Francia

17 | 2017

Hermetismo programático en la literatura rioplatense contemporánea (de 1980 a nuestros días)

\title{
Hermetismo programático en la literatura rioplatense contemporánea: avatares de lo legible
}

\section{Marta Inés Waldegaray}

\section{OpenEdition}

1 Journals

Electronic version

URL: http://journals.openedition.org/lirico/3821

DOI: $10.4000 /$ lirico.3821

ISSN: 2262-8339

Publisher

Réseau interuniversitaire d'étude des littératures contemporaines du Río de la Plata

\section{Electronic reference}

Marta Inés Waldegaray, « Hermetismo programático en la literatura rioplatense contemporánea: avatares de lo legible », Cuadernos LIRICO [En línea], 17 | 2017, Puesto en línea el 20 marzo 2018, consultado el 19 abril 2019. URL : http://journals.openedition.org/lirico/3821 ; DOI : 10.4000/ lirico.3821

This text was automatically generated on 19 April 2019

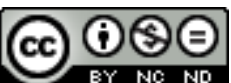

Cuadernos LIRICO está distribuido bajo una Licencia Creative Commons Atribución-NoComercialSinDerivar 4.0 Internacional. 


\title{
Hermetismo programático en la literatura rioplatense contemporánea: avatares de lo legible
}

\author{
Marta Inés Waldegaray
}

\section{Presentación}

$1 \quad$ Los artículos que se publican en este número dan cuenta de la reflexión y de los debates que en torno a la noción de legibilidad, hermetismo y programa escriturario en la literatura contemporánea de las últimas décadas tuvieron lugar durante los dos encuentros convocados conjuntamente por los equipos Li.Ri.Co. y A2IL ${ }^{1}$ de la Universidad de Reims Champagne-Ardenne, el 10 de diciembre de 2016 en la Universidad de Paris 8, y el 15 de marzo de 2017 en la URCA. La problemática de ambas jornadas se inscribe en la continuidad de las investigaciones que ambos grupos realizan en torno a la lectura literaria, y particularmente, en la continuidad de los trabajos iniciados por Vincent Jouve hacia finales de los años noventa en Reims sobre la noción de « ilegibilidad».

2 Muy frecuentemente abordada desde un ángulo de interés centrado en la recepción o en la lectura como práctica productiva de un texto, nos hemos interesado en ambas jornadas por formas de escritura que se constituyen desde una voluntad de prescindir de un sentido concebido como plenamente instalado, esto es: desde una voluntad de instalarse en una suerte de obstinación por adoptar operaciones de denegación que provocan constantes desplazamientos de la legibilidad. La tradición vanguardista argentina nos ha conducido a centrar nuestro interés en la noción de «hermetismo», concepto a menudo relacionado con lo que resulta ilegible y abierto a un amplio abanico de campos de análisis entrelazados: teóricos, operativos, ideológicos, temáticos. Sin embargo, los trabajos aquí publicados vislumbran una sutil brecha entre ambos conceptos, al conjeturar que en algunas producciones argentinas recientes ambas condiciones no son 
inevitablemente sinonímicas y que la declaración de ilegibilidad de un texto no es necesariamente un rasgo inherente de lo hermético (aunque a menudo uno y otro se entrelacen); ni lo hermético una subcategoría correspondiente a un gesto deseado de ilegibilidad, como parece proponerlo Vincent Jouve en su introducción a las Actas del Congreso que tuvo lugar en Reims en octubre de 1997:

L'illisibilité peut en effet être voulue (hermétisme, déstabilisation à visée idéologique, sacralisation d'une littérature pour initiés, expression de la singularité du moi) ou relative à un lectorat (dépourvu du savoir minimal postulé par le texte) et une époque (ne comprenant plus telle codification générique). ${ }^{2}$

La dimensión programática del hermetismo, tal como en este número se la aborda, se acerca más a una concepción de lo que la Literatura (el Arte de manera general) es o debería ser para algunos autores, y menos a un gesto « deseado » (« voulu »), en el sentido de calculado o pre-meditado. Los artículos tratan diversas facetas de esta heterodoxa singularidad que plantea lo hermético.

El número está compuesto por cuatro secciones: artículos (diez contribuciones); entrevistas (tres participaciones correspondientes a los escritores Amir Hamed, Sergio Chejfec y Pola Oloixarac, estudiados en los artículos); traducciones (cuatro trabajos realizados por Laura Gentilezza, Sonia Fernández Hoyos, Laura Corona y Valeria Grinberg $\mathrm{Pla}$, correspondientes a tres trabajos publicados originariamente en francés y uno en inglés sobre la temática relativa a los límites de la legibilidad); y finalmente, una sección de reseñas (que incluye dos notas de lectura elaboradas por Maximiliano Crespi y Andrea Carriquiry). A todos ellos agradecemos sus valiosos aportes y su interés, renovado en algunos de ellos, por la Revista. El número desea recordar a Amir Hamed, cuyo fallecimiento sobrevino el 20 de noviembre en Montevideo. Su inusitada, deslumbrante y prolífica obra no cesará de producir nuevos encuentros y nuevos lectores.

5 Pensar las formas de escritura que se erigen desde el rechazo de un sentido imaginado como pleno y desde el quebrantamiento del orden referencial supone interrogar tanto los niveles de (i-)legibilidad instaurados por la composición textual (códigos, motivos, dispositivos de escritura) como los procedimientos de contextualización que el lector realiza. Si bien nada es totalmente hermético, ni nada es enteramente transparente y legible, lo cierto es que la condición hermética de un texto obnubila su recepción e incita ejemplarmente a rehuir toda mecanización de la lectura. Si de lo que se trata es menos de comprender que de captar (o construir) condensaciones de sentido, la tradición vanguardista y rupturista de la literatura argentina es un terreno particularmente apto y acogedor para estos interrogantes. Fue en torno a la literatura rioplatense que se reflexionó durante la primera jornada desarrollada en Paris. La literatura contemporánea europea y latinoamericana estuvieron presentes en la jornada realizada en Reims.

\section{Las aladas sandalias de Hermes}

6 Dios mensajero, mediador y garante de la comunicación, Hermes es el dios de las sandalias aladas a quien se le atribuye la invención de la palabra y de la música, esto es : la creación de la comunicación etérea que, como un soplo, se esparce, se disgrega, se desgrana, se desintegra. Hermes es el portador de la palabra celestial. Representa el sentido que interrogado e indagado se esfuma en la voz y entre los vericuetos de la letra escrita. El sentido « hermético » es ese excedente irreductible que pone en evidencia la distinción entre lo semiótico y lo semántico ; es restancia ${ }^{3}$ o remanencia diseminada de 
significantes ; es sentido no apropiable que rechaza la lógica binaria de la representación, declinable como significante-significado, o presencia-ausencia, pares binarios que suponen la dificultad textual como resultado de una interferencia, de una claridad subyacente o latente obstruida por una formulación incomprensible. Estos pares binarios son apuntalados por un discernimiento cartesiano y negativo de la opacidad. En su ensayo acerca de la noción de dificultad, George Steiner teoriza al respecto :

What do we mean when we say : « this poem, or this passage in this poem is difficult ?» [...] What is signified by the pragmatic experience that a lexically constituted and grammatically organized semantic system can generate impenetrability and undecidabilities of sense? No coherent answer could be given outside a complete model, such as we do not have, of the relations between "thought " and speech, and outside a total epistemology, which again we do not have, of the congruence of speech-forms with a "prededent " body of intention, perception, and vocative impulse. In such a model « difficulty » would, presumably, be an interference-effect between underlying clarity and obstructed formulation. This, roughly, is the classical and Cartesian reading of opaqueness, a reading whose interference is necessarily negative. ${ }^{4}$

7 En este mismo sentido cuestionador de lo que se postula de manera ortodoxa como ilegible, desde una perspectiva didáctica y centrada en la enseñanza de textos literarios considerados como ilegibles en el ámbito universitario, es sugestivo el abordaje realizado por Annie Rouxel. La investigadora francesa relaciona la ilegibilidad principalmente con el olvido del horizonte referencial externo y menos con el desvío de un uso normativo del lenguaje. Rouxel propone que:

Si la subjectivité -et/ou la compétence- du lecteur sont déterminantes dans l'échec du procès de lecture, le texte qualifié d'illisible se présente sous des formes extrêmement diverses. On serait tenté de croire que le texte illisible par excellence est celui dont l'expression s'écarte le plus de la norme de lisibilité définie par la communication quotidienne : il se situerait non aux marges mais dans un au-delà des usages langagiers. Pourtant il n'en est rien : la conformité à l'usage quotidien du langage n'est pas gage de lisibilité.

Ainsi, qu'il s'agisse du déploiement verbal du texte surréaliste lié à l'écriture automatique ou de l'ascèse mallarméenne, ces textes, chacun à sa façon, « cèdent l'initiative aux mots ", créent des univers clos et suspendent la référence au réel. C'est donc sur ces aspects de la modernité poétique qu'il faut réfléchir avec les étudiants. ${ }^{5}$

8 Frente a la semiosis hermética, el razonamiento sólo parece poder surgir de una iluminación no discursiva. Umberto Eco propone que el pensamiento hermético es "práctica interpretativa del mundo y de los textos basada en la determinación de relaciones de simpatía que vinculan recíprocamente micro y macrocosmos $»^{6}$. Estas relaciones de simpatía sólo son posibles si se acepta el principio de no contradicción, ya que cuando los elementos contrarios entran en contacto se desintegra el principio de identidad, entra en crisis la idea de linealidad, cae la relación causa-efecto y sorprendentemente "tout se tient $»^{7}$. Así, -sostiene Eco- el pensamiento hermético " sustrae al lenguaje todo poder comunicativo. ${ }^{8}$

9 Los textos y autores estudiados comparten una dimensión programática (estratégica) tendiente a alterar los umbrales de la legibilidad, a interrogar la taxatividad de la oposición legible/ilegible. La impenetrabilidad del sentido es una forma extrema de la legibilidad $^{9}$; es un criterio que reviste experiencias diversas, pero que en definitiva, como lo apunta Craig Dworkin, consiste esencialmente en instaurar estrategias de escritura que desafían la gramática de recepción normativa de un texto. Escribe Dworkin: « All reading, 
of course, involves the reader's production of signification to some degree; the point is that such production is too often routine and disciplined by preestablished and inflexible protocols. $»^{10} \mathrm{El}$ gesto programático no es entendido en este volumen como necesariamente preceptivo, ni abordado como práctica deliberada y planificada, a la manera de un manifiesto positivista intentio auctoris - intentio operis, sino como experimentación que lleva al extremo la perplejidad del lector, reclama una alterlegibilidad ${ }^{11} \mathrm{y}$ construye una lectura paragramática que pone a prueba la capacidad de captar, conectar y construir reparos de sentido para erigir en definitiva una memoria desde la cual continuar leyendo. Sin ser preceptiva, la dimensión programática comprende certidumbres estructurantes sobre la propia producción ficcional y crítica. Tenazmente asentada, asume el riesgo de una escritura excesiva que se sabe inabordable o tratable en sus extremos; es allí en donde estos textos, que poseen a menudo una anómala elasticidad sintáctica, producen el enigma de su hermetismo. Lo que el crítico argentino Emmanuel Biset llama (con resonancia derrideana) « el dictado de la mano ». Lo cito : «Pero la ley de la escritura se ubica también en otro lado, en lo que se puede llamar un dictado de la mano. La ley aquí tiene la forma no de un imperativo, sino de una insistencia, de una obstinación. $»^{12}$. El investigador belga Michel Lisse también se interesa por la responsabilidad delegada a la mano en el acto de escribir. En la línea de los trabajos de Heidegger, Derrida y Jean-Luc Nancy sobre la ontología de la mano en su relación con el tocar, el ser, el pensamiento, la palabra y la escritura manuscrita, sostiene : « L'écriture et la lecture sont donc une adresse, mais une adresse qui demeure incertaine, comme le veut la loi du tact $[. .]. »{ }^{13}$. Siguiendo a Lisse, cabe preguntarse : ¿ cómo se lee, cómo se toca, un texto que nos obnubila y que parece querer expulsar al lector a la inconfortable situación de un "noli me legere», "noli me tangere»? Jean-Marc Talpin trata estos interrogantes sobre la experiencia corporal de la lectura de un texto que se nos escapa. " [L]e texte illisible fait violence au lecteur, violence qui passe dans l'expérience psychique et physique de la lecture. », apunta Talpin. Y agrega : «En effet, la lecture à haute ou basse voix, en tout cas, la lecture sonorisé [...] est utilisée par certains lecteurs comme par certains auteurs pour dépasser cet effet d'illisibilité. $»^{14}$. Decir y escuchar entonces para leer más o acaso mejor. En la propuesta de Talpin resuena el « oír entre líneas » de George Steiner y el conocido interrogante de Roland Barthes : «Ne vous est-il jamais arrivé, lisant un livre, de vous arrêter sans cesse dans votre lecture, non par désintérêt, mais au contraire par afflux d'idées, d'excitations, d'associations? En un mot, ne vous est-il pas arrivé de lire en levant la tête? ${ }^{15}$. Por cierto, el propósito del análisis de Talpin y de Barthes no es exactamente el mismo, pero lo que ambas captaciones de la lectura parecen tener en común es la constatación de que la escritura, convertida en desafiante peripecia material de encriptación, denegación o diferimiento de sentido, compromete el acto corporal del leer; envuelve el ritmo de la respiración, la tensión del cuerpo, la lectura en voz alta, el recitado en voz baja para intentar paliar lo que nos desconcierta o confunde.

\section{Facetas de la experiencia hermética}

La búsqueda de parámetros de lectura no convencionales es el desafío literario que propone una buena parte de la ficción narrativa de las últimas décadas creada por escritores como Daniel Guebel, Héctor Libertella, Luis Chitarroni, Sergio Chejfec, Carlos 
Gamerro, Amir Hamed, Pola Oloixarac. Y menos recientemente, aunque siempre vigentes, las ficciones de Juan José Saer, Luis Gusmán, César Aira.

El volumen propone diez estudios acerca de la ficción narrativa rioplatense de las últimas cuatro décadas en los cuales se reflexiona sobre las condiciones ideológicas y formales que rigen el aspecto hermético en sus producciones. Los textos analizados ponen en escena (porque lo dramatizan en sus temáticas o porque lo convierten en estilo) los límites de la interpretación, no solamente en el sentido de la negatividad radical mallarmeana o de la postergación infinita del acto interpretativo pleno y acabado, sino también en el sentido de un más allá de los límites convencionales de lo (de-)codificable. Comprensión de la experiencia, lectura crítica, adivinación, alucinaciones, sueños, traducción son algunas de las facetas desde las cuales la clausura de las claves interpretativas se manifesta y que los textos aquí estudiados problematizan. Lo oscuro, lo solapado, lo oculto, lo moroso son componentes esenciales de los avatares herméticos que los artículos de este número analizan. La conexión de efectos e informaciones parciales mediante el desvanecimiento de las categorías tempo-espaciales, lo fragmentario que aventaja al todo, la causalidad disgregada, la referencialidad particularmente elíptica e inestable, las líneas narrativas que se interrumpen y se multiplican continuamente, son los procedimientos más estudiados por los críticos de este volumen como causas productoras de una lectura reiteradamente interrumpida.

12 Los diez artículos que integran este volumen han sido agrupados en dos secciones. « Escribir denegando » es el eje articulador de la primera sección que reúne seis artículos. Se incluyen en ella los trabajos de : Julio Prieto, Graciela Villanueva, Marta Waldegaray, Camilo Bogoya, Benoît Coquil y Martín Arias. « Descifrando códigos » es el eje que federa la segunda, en la cual se agrupan los otros cuatro trabajos. Se trata de los ensayos de: Claudia Hammerschmidt, Edgardo Dobry, Mariela Blanco y Andrea Cobas Carral.

13 Julio Prieto explora en « De la ciega taquigrafía: la elusiva erudición de Luis Chitarroni » la poética de la opacidad en la escritura de Chitarroni y en particular en Peripecias del no. Diario de una novela inconclusa (2007). Prieto demuestra la manera en la que esta novela al decir atenuando y nombrar denegando reactiva tanto una específica tradición literaria argentina de poéticas de lo ilegible que abarca un amplio y diverso espectro desde Macedonio Fernández, Roberto Arlt o Felisberto Hernández hasta los hermanos Lamborghini, Copi, Perlongher, Pizarnik, Zelarrayán, Libertella, como también, las productivas ilegibilidades practicadas por autores que tienen un lugar relevante en la narrativa argentina contemporánea como Fogwill, Laiseca, Aira, Chejfec, Guebel, Cucurto o Bejerman. Son todas éstas expresiones de poéticas de errancia semiótica vinculadas con las prácticas del impedimento y de la reducción estética que transitan la modernidad artística y literaria. Notables prácticas de opacidades atravesadas por atisbos informativos, acopio de enigmas, vacilantes referencias, líneas narrativas interrumpidas y diferidas que estimulan y a la vez rehusan la interpretación, «nominaciones indefinidas que ponen en juego una específica dinámica de iluminación y ceguera, una suerte de parpadeo entre el deseo y la futilidad de leer », escribe brillantemente Julio Prieto.

Graciela Villanueva analiza en " "Cosas raras" : el juego del hermetismo en la ficción de César Aira » cómo desde una posición crítica heredera de las vanguardias históricas y opuesta a los principios de causalidad e identidad en la organización discursiva, Aira propone una escritura desde el margen del logocentrismo, del monologismo, de la lógica binaria, y también, del mercado y de la literatura comercial. Villanueva concentra su análisis de la obra airiana en un mecanismo determinante para la construcción de sus 
ficciones consistente en la (des)articulación de la relación entre el comienzo y el final del relato: "Para decirlo brevemente: si el comienzo de un texto narrativo abre interrogantes que su desarrollo y sobre todo su final acaban por responder, las novelitas de Aira dejan, en cambio, muchas preguntas sin respuesta y decepcionan a quien busque en las últimas páginas alguna clave que lo ayude a interpretar lo que acaba de leer.", escribe la crítica argentina. Este sentido deceptivo revela el principio constructivo de la discontinuidad (cambio de tema, de tono, de registro, de intertexto, de código), provoca perplejidad en el lector para quien las categorías y los códigos usuales con los que normalmente el lector se maneja no cesan de parecer inapropiados. Un trabajo juguetón y deceptivo que tiene mucho que ver con la concepción deleuziana de lo que para Aira debe ser la verdadera Literatura: sentido fundado en el sinsentido, sentido que no es ni fundamento ni origen, sentido concebido como permanente producción de sentido en el continuo de la superficie textual.

En « Fisuras de lo legible en Cielo 1/2 de Amir Hamed », Marta Waldegaray se interesa por la erudición densa e intrincada de esta extensa novela del escritor uruguayo ; una novela hermética en la medida en que su lectura parece destinada a iniciados en materia de dioses, cosmogonía, literaturas de diferentes épocas y latitudes. Pero hermética también, por la manera en que su funcionamiento narrativo obstaculiza el orden causal, vela su universo referencial y escamotea la voz narrativa. Se estudian en este artículo las operaciones dominantes de una escritura hipertrabajada consistente en una deriva errante hecha de injertos culturales y de cruces de materiales literarios de diferentes grados de nobleza que dejan cabos sueltos, interrogantes sin respuesta y cierres siempre provisorios que privan al lector de los puntos de apoyo necesarios para retener la actividad semiótica (desbordante) de la escritura de Hamed. En definitiva, una densa opacidad provocada en el plano narrativo por la apertura constante de claves de lectura que no siempre se clausuran; en el plano lingüístico, por la sintaxis enmarañada y el empleo de desconcertantes sintagmas y asociaciones léxicas; en el plano discursivo, por las rupturas de los registros discursivos (que van y vienen entre lo bajo, lo literario, lo mítico-religioso, lo coloquial) y la ausencia de ortodoxas marcas enunciativas. Este sistema calidoscópico (que desplaza permanentemente los centros narrativos, gramaticales, sintácticos) comporta escasos momentos de reposo para el lector. La noción de hermetismo es abordada aquí entonces como legibilidad en suspenso que atiza la performance del lector. La condición hermética de la escritura de Hamed parece tener un agudo sentido protector del arte verbal, concebido como ámbito y experiencia de lo Absoluto literario.

Camilo Bogoya vuelve sobre la obra de Juan José Saer en «La custodia del sentido. Una lectura de Nadie nada nunca » para recordar la defensa que el escritor argentino hace de una literatura fundamentada en la dificultad, la incertidumbre de la percepción, la tensión entre una obra orgánica y a la vez fragmentada, un proyecto narrativo emparentado con lo que puede denominarse como rasgos herméticos saerianos consistente en descentrar los valores tradicionales de legibilidad y progresión narrativa, para ofrecer opacidad, estancamiento narrativo en un presente dilatado, fragmentación continua y multiplicación de unidades narrativas. Bogoya escoge como obra central para su análisis la novela Nadie nada nunca (1980) por el lugar culminante que este texto tiene en el interior de la obra saeriana marcada como se sabe por la indagación formal y su relación dramatizada con el sentido oscuro. Este sentido se encuentra, según lo propone Bogoya, voluntariamente « encapsulado » ya que lo que expone el programa literario de 
Saer es una custodia del sentido que tiene que ver formalmente con las postergaciones e interrupciones del relato, las tentativas de mantener la atención del lector y la defensa de valores literarios que el autor expone en primera línea tanto en sus ficciones como en el pensamiento teórico manifestado en sus ensayos. Bogoya también vuelve a algunos de ellos con la intención de desentrañar el peso del silencio en los textos somnolientos de Saer, un silencio que encauza el sentido hacia los umbrales del hermetismo. En Nadie nada nunca entonces, el hermetismo es entendido como negatividad productiva, sentido retenido en la intemperie oscura, aletargada y compleja de la narración. En la impugnación de los valores literarios de sus contemporáneos y en la resistencia a la homogenización cultural, Bogoya lee la dimensión programática del hermetismo saeriano.

Benoît Coquil elucida en " "Complejizar lo existente" : resistencias del sentido en la obra de Sergio Chejfec » las modalidades y alcances del hermetismo en textos de ficción como Moral (1990), El aire (1992), Boca de lobo (1999), Los planetas (1999), Los incompletos (2004), Lenta biografía (2007), en textos ensayísticos como últimas noticias de la escritura (2015) y en algunas publicaciones periodísticas. Coquil emprende su análisis asentando que el hermetismo en Chejfec es el correlato de una doble experiencia estética : por un lado, en los años 90, el rechazo generacional a una literatura considerada demasiado transparente y normativa ; por otro lado, la ficcionalización de cuestiones relativas a su propia historia familiar y a la identidad judía. Partiendo de la constatación de que el desciframiento impracticable de lo real es una constante en la obra de Chejfec, Coquil proporciona una tipología de rasgos formales que contribuyen de manera recurrente a la fractura y deriva de la linealidad narrativa, como: la descripción que dilata el fluir de lo contado, el modo reiterativo, la estructura circular, los estancamientos del relato. El hermetismo resulta dramatizado cuando Chejfec pone en escena personajes sumergidos en la perplejidad suscitada por la progresiva ilegibilidad de su entorno, un estado de letargo y de estupefacción en el cual la realidad aparece inesperadamente extraña para quien la contempla; personajes sumergidos en la oscuridad; o bien, personajes que vagabundean. Coquil lee en el ritmo lento de la sintaxis de los textos de Chejfec y en sus personajes morosos una melancolía asociable al hermetismo, que impone a su lector una somnolienta errancia textual. La narrativa de Chejfec aborda temas políticos, memoriales e identitarios (como la memoria de la Shoah, del Proceso, del exilio, de la identidad argentina y judía) por caminos oscuros y desviados. El hermetismo en Chejfec, concluye Coquil, no es sinónimo de clausura del sentido, de intransitividad, ni de autocomplacencia erudita. Su hermetismo reside en una programación de lo ilegible que apunta a la performance del lector. En su convicción acerca de la imposibilidad de la representación de lo real, la negatividad productiva de Chejfec radicaliza el trabajo literario de Saer.

18 Finalmente en " "Hermetismo": un muestrario », último artículo de la primera sección, Martín Arias reflexiona acerca de la noción que federa el volumen a partir de un corpus heterogéneo de textos. Sobre la base de un breve muestrario de apariciones del concepto de "hermetismo " y del adjetivo "hermético » en la literatura y en la crítica literaria argentinas producidas entre 2003 y 2016, Arias explora los usos de ambos términos en trabajos críticos como el ensayo de Tamara Kamenszain Una intimidad inofensiva. Los que escriben con lo que hay (2016), la reseña de Martín Kohan dedicada a Los sospechados (2002), de Milita Molina, y en dos obras de Héctor Libertella: El árbol de Saussure (2000) y Ensayos o pruebas sobre una red hermética (1990). Aborda las dos primeras obras de Milita Molina, Fina 
voluntad (compuesto por las nouvelles «El visitante » $\mathrm{y}$ « Greta Krull », de 1993) y la novela Una cortesía (1998). Arias indaga cómo unos y otros escritores (que coinciden en practicar tanto el ejercicio de la literatura como el de la crítica) coinciden en considerar lo hermético como una zona resistente o cerrada a la interpretación (a la manera del trobar clus medieval), como una aversión a volver comprensible lo que es denso, todo lo cual es relacionable según Arias con otro postulado sustancial y contundente: el que -siguiendo a Libertella- reza que la literatura no es un pensamiento; o el que -recordando a Eduardo Wilde- sugiere que la literatura es (un) pensamiento contrahecho o tortura del pensamiento. El hermetismo nombra un desequilibrio en la tensión sígnica inherente a la literatura; designa una alteración en el sistema semiótico desertado por el pensamiento, concluye Arias.

El trabajo de la crítica alemana Claudia Hammerschmidt inaugura la segunda sección. En «La dicotomía de lo legible e ilegible en la literatura argentina contemporánea, o El lado oscuro de Carlos Gamerro ", Hammerschmidt lee lo ilegible en la obra de Gamerro y especialmente en su novela Las Islas (1998). Propone en su estudio que lo ilegible a finales del siglo XX y a principios del XXI en la literatura contemporánea argentina ha intensificado programáticamente el vacío constitutivo del lenguaje moderno. La lectura atenta que de la obra de Gamerro hace Hammerschmidt le permite sostener que la experiencia de los límites que la escritura de Gamerro expone es el correlato formal y moderno de las víctimas históricas de los excesos cometidos por el estado argentino durante la dictadura. La crítica alemana se basa en los posicionamientos teóricos de Roland Barthes, Michel Foucault y Paul de Man para quienes lo ilegible es el lado subterráneo, autónomo e incontrolable de la literatura moderna; desde este lado oscuro del lenguaje literario, los crímenes e injusticias resultan visibilizados. Constatar entonces la presencia de lo ilegible en la producción literaria argentina a fines del siglo XX, podría implicar -según lo sugiere Hammerschmidt- un contraste entre una primera generación posdictatorial que tomó distancia de esta zona oscura y adoptó un discurso de registro realista refractario a las sutiles alusiones, y otra generación más contemporánea que, saturada del discurso de los hechos, desconfió de la pretensión de legibilidad de una expresión más explícita y se orientó hacia ese otro lado del lenguaje literario que asume lo indecible como principio inherente a lo literario. Basándose en la literatura de Gamerro, Hammerschmidt lee este giro como una tendencia que, hacia finales del siglo XX y a principios del XXI, aviva hermética y programáticamente el vacío constitutivo del lenguaje moderno para desvelar la brutalidad de la Argentina neoliberal.

Edgardo Dobry indaga en su artículo «Pizarnik y Gelman ante la ley inaccesible » la relación que ambos poetas argentinos, nacidos en la década de 1930, tienen con el judaísmo. Sobre la base del concepto de "rebelión de los hijos » en el universo familiar judío trabajado por Stephane Mosès en L'ange de l'histoire (1992) y su aplicación a la situación en el mundo judío centroeuropeo entre finales del siglo XIX y principios del XX, el interesante ángulo de análisis propuesto por Dobry en su artículo consiste en una lectura de la ilegibilidad de la tradición judía para ambos poetas argentinos, en la imposibilidad compartida de acceder a la Ley de su pasado cultural. Según lo propone Dobry para ambos autores, y remontándose a $E l$ proceso de Franz Kafka, escritor a quien toma como modelo para la problemática que plantea, la perturbación de la Ley es en ambos escritores argentinos un interrogante poético y existencial. Existe en ellos la certidumbre de que la Ley que estructuraba la experiencia de sus antepasados sigue presente, pero de una manera inasequible en el mundo definitivamente secularizado en el 
que se encuentran. La originalidad de la propuesta ofrecida por Dobry en su artículo pone de realce la obra tardía de ambos escritores que practicaron, por cierto, estéticas muy distintas. La « cuestión judía » irrumpe en el tramo final de sus obras vinculada a la figura parental. Asociada a la figura del padre en Pizarnik, el interrogante parental se vuelve urgente a la muerte de éste; asociada a la figura materna en Gelman, la muerte de la madre desencadena en él una indagación bastante equivalente. Desde la línea de análisis planteada por Mosès, Dobry elucida cómo Pizarnik y Gelman, ambos hijos argentinos de inmigrantes judíos askenazis, ofrecen una condición particular: el de querer ser argentinos e inscribirse en la vida social, política, cultural del país en el que nacieron sin desentenderse por esto de su condición judía de origen. Ambos revelan una vocación de regreso al judaísmo, aunque vuelto interrogante, puesto que lo hacen con la certeza de haber perdido su clave de lectura y de que aquella Ley sólo puede ser evocada y no plenamente poseída.

21 Mariela Blanco analiza en "Lo legible/ilegible en la narrativa de Pola Oloixarac » los procedimientos discursivos que conforman la sintaxis hermética en las dos novelas que hasta el momento ha publicado la joven narradora argentina Pola Oloixarac: Las teorías salvajes (2008) y Las constelaciones oscuras (2015). La hipótesis de Blanco reside en que la problemática de lo legible es uno de los ejes centrales de la narrativa de Oloixarac, en la medida en que los textos estudiados instalan la pregunta por las condiciones de legibilidad de determinadas textualidades en determinados contextos históricos. Lo « hermético » no es para Blanco sinónimo de «ilegible». La crítica argentina centra su análisis en los procedimientos de montaje como mecanismo clave para erigir una sintaxis hermética que, al yuxtaponer y dislocar distintas historias, vuelve más legible los relatos de la violencia estatal. La dimensión programática de la tensión legible/ilegible expone en Oloxairac el funcionamiento de la sintaxis del poder. La sintaxis emerge en sus textos temática y programáticamente como una estructura ordenadora a la que hay que dislocar. Finalmente, Blanco traza un vínculo genealógico con la escritura de Jorge Luis Borges a partir de la distopía como recurso que permite representar alegóricamente y a la vez reflexionar sobre el orden social desde la literatura.

El volumen se cierra con el estudio de Andrea Cobas Carral quien en «Decir lo indecible. Violencia política y hermetismo en la escritura de Luis Gusmán» analiza los procedimientos mediantes los cuales Gusmán lleva a la literatura la experiencia de la aniquilación y la censura vinculando sentidos que expresan lo que parece indecible en el contexto de dolor y de censura de la dictadura argentina. Centrándose en un estudio contrastivo de las dos versiones de la novela En el corazón de junio (1983 y 1999), pero apelando también a El frasquito (1973) y a Villa (1995), Cobas Carral aborda su propósito teniendo en cuenta dos momentos claramente recortados de la labor literaria de Gusmán : un primer momento que abarca los años $70 \mathrm{y}$ se extiende hasta mediados de los 80 , en el cual sus primeros textos y ficciones manifiestan un franco desafío a la codificación realista de lo inteligible. Un segundo momento, ubicado hacia mediados de los 80 , en el cual el autor inicia un progresivo alejamiento de las concepciones estéticas dominantes en su etapa anterior para indagar la referencialidad política a través de formas de representación más cercanas al realismo. Esta transformación acompaña un cambio de época que es también para Gusmán la ocasión de indagar otros modos de practicar la labor literaria y otros modos de concebir la función de la literatura en el contexto de la democracia. Establecida esta problemática interna en la obra del autor, Cobas Carral emprende el estudio de la primera etapa puesto que en ella se expone como rasgo 
dominante el problema de la construcción de sentido. El hermetismo es en ese primer momento de las ficciones de Gusmán, según Cobas Carral, « una problemática poética que la ficción construye como tema y que permite, por extensión, significar la violencia y decir aquello que no podría ser dicho de otro modo ».

Lejos de pretender marcar el perímetro de un concepto, los aportes de este volumen reúnen análisis y reflexiones que ponen en evidencia el carácter fluctuante de la noción de « hermetismo programático », las diversas facetas de este ejercicio de alter-legibilidad. Erudición ecléctica, hiperliteratura, extravío semiótico, quiebres narrativos, textos literarios o culturales que encapsulan claves y misterios, retórica barroca (entendida como expresión semiótica), pathos neobarroco (que a diferencia de lo barroco no admite la glosa), verdad indescifrable, códigos caducos, zonas de ceguera textual, denegación y diferimiento del sentido, sentido sin fondo, sinsentido que rebate tanto la ausencia de sentido como la creencia en un sentido pleno y originario... todas estas perspectivas, no excluyentes unas de otras, constituyen los avatares de las experiencias herméticas tratadas en este volumen.

\section{NOTES}

1. El seminario « Approches Interdisciplinaires et Internationales de la Lecture » (A2IL) trabaja de manera transversal entre dos equipos de investigación de la Universidad de Reims : el Centre Interdisciplinaire de Recherche sur les Langues Et la Pensée (CIRLEP, EA4299) y el Centre de Recherche Interdisciplinaire sur les Modèles Esthétiques et Littéraires (CRIMEL, EA3311). El segundo encuentro se realizó en Reims, el 15 de marzo de 2017. En esta oportunidad se abordaron diversas propuestas de escritores europeos y latinoamericanos contemporáneos. Las actas de la jornada que tuvo lugar en la Universidad de Reims serán editadas por la colección EPURE y publicadas en el primer semestre de 2018.

2. Vincent Jouve (dir.), Introducción a La lecture littéraire. Revue de Recherche sur la Lecture des Textes Littéraires, n³: L'illisible, janvier 1999, Klincksieck, p.7-12; p. 7. Actas del coloquio organizado en la Universidad de Reims, 21-23 de octubre de 1997.

3. Jacques Derrida, La dissémination, Paris, Seuil, 1993, p. 59.

4. George Steiner, «On Difficulty », The Journal of Aesthetics and Art Criticism, vol. 36, n 3 : Critical Interpretation, Spring 1978, p. 263-276; p. 263. Destacado en el original.

5. Annie Rouxel, « La lecture littéraire, un jeu avec l'illisible », in: Vincent Jouve (dir.), La lecture littéraire. Revue de Recherche sur la Lecture des Textes Littéraires, $\mathrm{n}^{\circ} 3$ : L'illisible, op.cit., p. 135-142 ; p. 135 et 138 respectivement. La cita introducida por Rouxel pertenece a Stéphane Mallarmé, Crise de vers, NRF, Gallimard, p. 248. Se incluye en nuestro volumen la traducción en español del artículo de Rouxel, realizada por Laura Corona.

6. Umberto Eco, Los límites de la interpretación, Barcelona, Editorial Lumen, 1992, p. 53. Edición original publicada en italiano, Milán 1990.

7. Ibid. p. 53. Sobre el secreto iniciático en la cultura griega del siglo II y la concepción de la verdad como aquello que no se dice o se dice oscuramente, asociable con la idea de profundidad, ver especialmente « Hermes », p. 51-52.

8. Ibid., p. 54. 
9. Jean-Marie Gleize, « Obscurément », in : Bénédicte Gorrillot et Alain Lescart (comp.), L'illisibilité en questions, Lille,

Presses Universitaires du Septentrion, coll. «Littératures », 2014, p. 37-41 ; p. 37.

10. Craig Douglas Dworkin, Reading the illegible, Evanston-Illinois, Northwestern University Press, 2003, p.11. Las primeras treinta páginas de este denso y conceptuoso ensayo se incluyen en el presente volumen traducidas por Valeria Grinberg Pla.

11. Es el término propuesto por Bénedicte Gorrillot en el volumen L'illisibilité en questions (op. cit).

12. Emmanuel Biset, prefacio a Gabriela Milone (comp.), La obstinación de la escritura, Córdoba, Postales Japonesas Editora, 2013, p. 5-12 ; p. 7.

13. Michel Lisse, "Lire, toucher : d'une main à l'autre ", in : Jean-François Chassay y Bertrand Gervais [editores], Paroles, textes et images. Formes et pouvoirs de l'imaginaire, Université du Québec à Montréal, Centre de Recherche sur le texte er l'image, coll. «Figura », n¹9, vol. 2, p. 157-168; p. 165. Artículo incluido en este número, en español, traducido por Laura Gentileza.

14. Jean-Marc Talpin, «L'illisible, le corps ", in: Vincent Jouve (dir.) La lecture littéraire. Revue de Recherche sur la Lecture des Textes Littéraires, n³ : L'illisible, op.cit., p.195-204; p. 196. Artículo incluido en español en este número, traducido por Sonia Fernández Hoyos.

15. Roland Barthes, Euvres complètes, éd. Eric Marty, Paris, Seuil, 1993-1995. « Ecrire la lecture », p. 961. También en Le Bruissement de la langue, Paris, Éditions du Seuil, 1984, p. 34.

\section{AUTHOR}

\section{MARTA INÉS WALDEGARAY}

Univesité de Reims Champagne-Ardenne

CIRLEP (EA 4299)

marta-ines.waldegaray@univ-reims.fr 